\title{
Distribuição espacial das mortes atribuíveis ao uso de álcool no Brasil
}

\section{Spatial distribution of deaths attributable to alcohol use in Brazil}

\author{
Marilane Vilela Marques ${ }^{1}\left(\mathbb{D}\right.$, Danyllo do Nascimento Silva Junior ${ }^{2}$ (D), Emelynne Gabrielly de Oliveira Santos ${ }^{3}$ 으, Samara Sybelle \\ de Araújo Nobre Santos ${ }^{4}$ (10), Sandra Mara Bezerra das Neves ${ }^{5}$ (1) , Ana Edimilda Amador ${ }^{6}$ (1) \\ 1. Especialista em Análise de Situação de Saúde pela Universidade Federal de Goiás (UFG), Goiânia, GO, Brasil. 2. Mestre em Saúde Coletiva pela Universidade \\ Federal do Rio Grande do Norte (UFRN), Natal, RN, Brasil. 3. Doutoranda pelo Programa de Saúde Coletiva pela Universidade Federal do Rio Grande do Norte \\ (UFRN), Natal, RN, Brasil. 4. Especialista em Gestão do Trabalho e da Educação na Saúde pela Universidade Federal do Rio Grande do Norte (UFRN), Natal, \\ RN, Brasil. 5. Especialista em Saúde Pública e Serviço Social pelo Centro Universitário Facex (UniFacex) Natal, RN, Brasil. 6. Doutoranda em Demografia pela \\ Universidade Federal do Rio Grande do Norte (UFRN), Natal, RN, Brasil.
}

\section{Resumo}

Objetivo: analisar a distribuição espacial das mortes atribuíveis ao uso de álcool no Brasil. Métodos: estudo epidemiológico descritivo e analítico nos 5.570 municípios do Brasil, no período de 2012 a 2016. Analisaram-se a distribuição espacial, a intensidade e a significância por meio do índice de Moran Global, MoranMap, LisaMap e BoxMap. Resultados: no período estudado, houve 33.168 óbitos atribuíveis ao uso de álcool. Desses, foi observada uma maior proporção de óbitos para o sexo masculino nas faixas entre 40 a 59 anos. Os municípios que apresentaram as maiores taxas médias de mortalidade padronizadas por 100 mil habitantes foram Mata - RS $(43,19)$, Pendências - RN $(40,74)$, Uru - SP $(36,43)$, Senhora do Porto - MG $(35,77)$, Novo Alegre - TO $(34,85)$, Arantina - MG $(33,14)$ e Catuji - MG $(32,71)$. 0 valor do Índice de Moran Global foi positivo e com significância estatística (p-valor=0,01). Evidenciou-se formação de clúster de alto/alto em municípios das regiões nordeste, sudeste e centro-oeste, enquanto foi verificada a presença de clúster de baixo/baixo nas regiões norte e sul do país. Conclusões: existe, no Brasil, um padrão de dependência espacial na distribuição das taxas de mortalidade atribuíveis ao uso do álcool.

Palavras-chave: Mortalidade. Álcool. Distribuição espacial. Dependência espacial.

\begin{abstract}
Objective: to analyze cesarean section rates in Santa Catarina and its association with source of payment for childbirth. Methods: Cross-sectional study based on the Information System of Live Births of Santa Catarina (SINASC/SC), 2012. The global and specific cesarean rates were calculated according to the SCR groups, in addition to the excess of cesarean sections due to the Proportional Attributable Risk (RAP). Differences in birth proportions and cesarean section in each group, according to the form of payment, were analyzed by the chi-square test at $5 \%$ significance level. Results: The overall cesarean section rate was $60.7 \%$, being $88.9 \%$ in the private system and $45.7 \%$ in the public one. Groups 1,4 and 5 of RCS were the ones with the greatest impact on cesarean overall rates. In the private sector, attention was drawn to the low use of induction, with more than $65 \%$ of deliveries occurring electively, in addition to the high concentration of multiparous women with previous cesarean section. Conclusions: High rates among primiparous women anticipate the cumulative effect of previous cesarean section in multiparous women. The large proportion of elective cesareans sections at the expense of active labor management, especially in the private sector, points to a large number of cesarean sections performed without medical indications and concerns due to potential adverse effects on mothers and newborns.
\end{abstract}

Keywords: Mortality. Alcohol. Spatial distribution. Spatial dependence.

\section{INTRODUÇÃO}

Os transtornos relacionados ao uso de álcool (TRA) apresentam alta prevalência no Brasil e no mundo, pois afetam 3,6\% da população mundial com idade entre 15 e 64 anos. No ano 2010, o uso de álcool era o quinto principal fator de risco para carga de doença no mundo; no Brasil, o terceiro fator de risco ${ }^{1}$.

Além da elevada morbidade e dos riscos sociais, estima-se que o uso abusivo do álcool acarrete 2,5 milhões de mortes a cada ano. Causas externas de morte, especialmente as decorrentes de acidentes de trânsito e agressões são associadas ao uso do álcool ${ }^{2}$.
O uso do álcool é um fator de risco e deve ser considerado como causa evitável para mortes prematuras ${ }^{3}$. O álcool é também a droga psicoativa mais usada na maioria dos países, tanto para a festividade como para esquecer a tristeza, pois seu consumo pode produzir vários danos biopsicossociais ${ }^{4}$. E sendo considerada uma droga amplamente consumida, classificado como uma droga lícita a qual favorece a sociabilidade e a integração entre os indivíduos ${ }^{5}$.

Segundo a Organização Mundial de Saúde (OMS), por ano, estima-se que ocorrem 3,3 milhões de mortes no mundo 
atribuíveis ao uso nocivo do álcool, o que representa 5,9\% da totalidade desse evento. No cenário mundial, indivíduos, com idade acima de 15 anos, bebem, em média, 6,2 litros de álcool puro por ano, o que representa 13,5 gramas de álcool puro por dia. Já no Brasil, o consumo do álcool é mais que o dobro da média mundial, sendo de 15,1 litros puros por ano, estando o País com taxas superiores a mais de 140 países $^{6}$.

Assim, o alcoolismo é um grave problema de saúde pública, que gera consequências drásticas tanto para o indivíduo que faz uso, como para familiares e amigos ${ }^{7}$. Ainda é apontado como sendo um fator de risco para o consumo e experimentação de outras drogas, outrossim, para a ocorrência de morbimortalidade por doenças crônicas não transmissíveis (DCNT) e por causas externas ${ }^{8}$.

O consumo excessivo de álcool também está relacionado a dez vezes o risco de mortalidade por cirrose hepática e desordens mentais, sete vezes o risco de injúria fatal e duas vezes o risco cardiovascular e morte por neoplasias ${ }^{9}$.

No ano de $2012,5,1 \%$ da carga global de doenças foi atribuível ao consumo do álcool, o que equivale a 139 milhões de anos de vida ajustados por incapacidade ${ }^{10}$. Sendo, então, um problema de ordem social, podendo causar danos extremos à saúde ou afetar o ambiente familiar, social, bem como as condições de trabalho ${ }^{11}$.

No Brasil, iniciativas por meio de políticas públicas tem buscado atenuar os efeitos deletérios do uso abusivo do álcool e de outras drogas. Nesse sentido em 2004, mediante a Portaria no 2.197, foi instituída a Política Nacional do Ministério da Saúde para Atenção Integral aos Usuários de Álcool e outras Drogas.12 Em 2007, a Política Nacional sobre o Álcool, por meio do decreto $n$ ㅇ 6.117, o qual dispõe sobre as medidas para redução do uso indevido de álcool e sua associação com a violência e criminalidade ${ }^{13}$.

Outras Políticas de Saúde Pública do Brasil foram implantadas, como o Plano Emergencial de Ampliação do Acesso ao Tratamento e Prevenção de Álcool e outras Drogas ${ }^{14}$, a Rede de Atenção Psicossocial para pessoas com sofrimento ou transtorno mental e com necessidades decorrentes do uso de crack, álcool e outras drogas ${ }^{15}$. O Plano de Ações Estratégicas para o Enfrentamento das DCNT no Brasil (2011 a 2022) que traz como meta a redução da prevalência de consumo nocivo de álcool, de 18\% (2011) para 12\% em (2022) ${ }^{16}$.

No Brasil e no mundo, o uso e o abuso de álcool e/ou outras drogas têm aumentado de forma constante, entre indivíduos de todas as camadas sociais, principalmente, entre os jovens. $E$ como problema de saúde pública merece muita atenção devido aos seus determinantes e condicionantes culturais, ético-legais, políticos, econômicos e tecnológicos, gerando impacto sobre o indivíduo, a família e a sociedade ${ }^{17}$.

Assim, é necessário pensar a relação entre consumo de álcool e qualidade de vida, pois os comportamentos associados ao consumo do álcool influenciam aspectos de saúde, e o estilo de vida das pessoas, muitas vezes, torna-se patológico, já que o indivíduo não consegue enfrentar o vício, ocorrendo recaídas, gerando mais danos à sua saúde ${ }^{18}$.

Neste sentido, as estratégias de Redução de Danos (RD) em consonância com a promoção dos direitos humanos devem ser desenvolvidas para ofertar prevenção e cuidado aos problemas relacionados ao uso de álcool e outras drogas em redes de apoio, cuidado e proteção, preservando-se a identidade e a liberdade na decisão do usuário, não se impondo a abstinência do uso de drogas como requisito para o acesso ao tratamento ${ }^{19}$.

A dependência do álcool pode afetar o convívio familiar, gerando implicações psicossociais e sobrecarga financeira para manter o uso, e isso repercute no relacionamento familiar e causa diferentes prejuízos para a família, que vão além de perdas financeiras ${ }^{20}$.

As consequências do uso de álcool também oneram a sociedade, de forma direta e indireta, potencializando os custos em hospitais e outros dispositivos do sistema de saúde, sistema judiciário, previdenciário, perda de produtividade do trabalho, absenteísmo, desemprego, entre outros ${ }^{21}$.

A avaliação do custo social relacionada ao álcool demonstra que o ambiente social no qual o álcool é consumido, conforme sua estruturação econômica e regras de convívio determinam diversos matizes de inserção do consumo alcoólico, ao mesmo tempo em que é diretamente influenciado pelos padrões de uso $^{22}$.

Diante do exposto, é importante conhecer a mortalidade atribuível ao uso do álcool nos diferentes municípios brasileiros, tendo em vista a diversidade cultural e socioeconômica no país. Assim, o objetivo deste estudo é analisar a distribuição espacial da mortalidade atribuível ao uso de álcool no Brasil.

\section{MÉTODOS}

Trata-se de um estudo ecológico que avaliou os óbitos decorrentes de transtornos mentais e comportamentais devido ao uso de álcool registrados no Brasil. Foram utilizados dados do Sistema de Informações sobre Mortalidade (SIM) do Ministério da Saúde (MS) sobre os óbitos ocorridos no período de 2012 a 2016 em residentes no Brasil, por meio da 10a Revisão da Classificação Internacional de Doenças e Problemas Relacionados à Saúde (CID-10), segundo a categoria F10.

Foram calculadas as Taxas Médias de Mortalidade Padronizada (TMP) para os municípios brasileiros para o período considerado no estudo. As taxas foram padronizadas, utilizando o método direto de padronização, sendo a população padrão estimada para o Brasil, no ano do meio do período (2014). Foi realizada a caracterização dos óbitos por sexo, faixa etária, estado civil, local de ocorrência. 
Para análise da estatística espacial de Moran Global e Local, utilizaram-se as Taxas médias de Mortalidade Padronizada (TMP) para o período de 2012 a 2016. A análise de dependência espacial foi executada, utilizando-se o índice de Moran Global que estima a autocorrelação espacial, podendo variar entre -1 e +1 , além de fornecer a significância estatística (valor de $p$ ). Foi construído o MoranMap relativo às taxas de mortalidade, apresentando apenas aqueles clusters com valor de $p<0,05$. Para a validação do Índice de Moran Global, foi utilizado o teste de permutação aleatória, com 99 permutações ${ }^{23}$. Para a produção dos mapas temáticos e o cálculo do Índice de Moran Global e Local, foi utilizado o software Terraview 4.2.2.

Um aspecto fundamental da análise exploratória espacial é a caracterização da dependência espacial, mostrando como os valores estão correlacionados no espaço. Nesse contexto, uma das funções utilizadas para estimar quanto ao valor observado de um atributo em uma região é dependente dos valores dessa mesma variável nas localizações vizinhas é autocorrelação espacial $^{24}$.
Essa pesquisa utilizou dados secundários disponíveis em sites oficiais do Ministério da Saúde do Brasil, sem identificação de sujeitos, sendo dispensado de apreciação em comitê de ética em pesquisa, em conformidade com a Resolução 466/2012 do Conselho Nacional de Saúde.

\section{RESULTADOS}

De acordo com os dados do Sistema de Informações sobre Mortalidade, no período de 2012 a 2016, foram registrados 33.168 óbitos atribuíveis ao uso de álcool no Brasil. Dos óbitos, $29.825(89,92 \%)$ são do sexo masculino. Por faixa etária, destacam-se as faixas com idade entre 40 a 59 anos com 18.705 óbitos (56,39\%); de 60 anos e mais com 8.585 óbitos $(25,88 \%)$. Ao analisar o estrato raça/cor, evidenciou-se que $60,17 \%$ são para pretos e pardos, enquanto $34,33 \%$ para brancos e amarelos. Por escolaridade, 28,04\% estudaram de 1 a 3 anos, e $1,14 \%$ estudaram 12 anos e mais. Por estado civil, com 48,22\% encontram-se os solteiros. Em relação ao local de ocorrência do óbito, $43,64 \%$ ocorreram no hospital seguido pelos ocorridos no domicílio com $42,66 \%$ (tabela 1 ).

Tabela 1. Caracterização dos óbitos atribuíveis ao uso de álcool no período de 2012 a 2016. Brasil, 2019.

\begin{tabular}{|c|c|c|c|}
\hline Variável & Categoria & 2012 a 2016 & $\%$ \\
\hline \multirow[t]{2}{*}{ Sexo } & Masculino & 29,825 & 89,92 \\
\hline & Feminino & 3,343 & 10,08 \\
\hline \multirow[t]{6}{*}{ Faixa Etária } & $<1$ a 14 anos & 3 & 0,01 \\
\hline & 15 a 19 anos & 49 & 0,15 \\
\hline & 20 a 39 anos & 5,734 & 17,29 \\
\hline & 40 a 59 anos & 18,705 & 56,39 \\
\hline & 60 anos e mais & 8,585 & 25,88 \\
\hline & Idade ignorado & 92 & 0,28 \\
\hline \multirow[t]{4}{*}{ Raça/ Cor } & Branca + Amarela & 11,385 & 34,33 \\
\hline & Preta + Parda & 19,956 & 60,17 \\
\hline & Indígena & 118 & 0,36 \\
\hline & Ignorado & 1,709 & 5,15 \\
\hline \multirow[t]{6}{*}{ Escolaridade } & Nenhuma & 5,505 & 16,60 \\
\hline & 1 a 3 anos & 9,300 & 28,04 \\
\hline & 4 a 7 anos & 7,274 & 21,93 \\
\hline & 8 a 11 anos & 2,991 & 9,02 \\
\hline & 12 anos e mais & 379 & 1,14 \\
\hline & Ignorado & 7,719 & 23,27 \\
\hline \multirow[t]{6}{*}{ Estado civil } & Solteiro & 15,994 & 48,22 \\
\hline & Casado & 7,438 & 22,43 \\
\hline & Viúvo & 1,821 & 5,49 \\
\hline & Separado judicialmente & 2,936 & 8,85 \\
\hline & Outro & 1,317 & 3,97 \\
\hline & Ignorado & 3,662 & 11,04 \\
\hline
\end{tabular}




\begin{tabular}{llrr}
\hline Variável & Categoria & 2012 a 2016 & \% \\
\hline Local de ocorrência & Hospital & 14,473 & 43,64 \\
& Outro estabelecimento de saúde & 1,958 & 5,90 \\
& Domicílio & 14,150 & 42,66 \\
& Via pública & 1,249 & 3,77 \\
& Outros & 1,278 & 3,85 \\
& Ignorado & 60 & 0,18 \\
\hline
\end{tabular}

Fonte: Datasus, Sistema de Informação sobre Mortalidade - SIM.

Os municípios que apresentaram as maiores taxas médias de mortalidade padronizadas foram Mata - RS (43,19 óbitos/100 mil hab.), Pendências - RN (40,74 óbitos/100 mil hab.), Uru SP $(36,43$ óbitos/100 mil hab.), Senhora do Porto - MG $(35,77$ óbitos/100 mil hab.), Novo Alegre - TO (34,85 óbitos/100 mil hab.), Arantina - MG (33,14 óbitos/100 mil hab.) e Catuji - MG $(32,71$ óbitos/100 mil hab.) (figura 1).

Figura 1. Espacialização da taxa média de mortalidade padronizada, atribuível ao uso do álcool para o período de 2012 a 2016. Brasil, 2019.

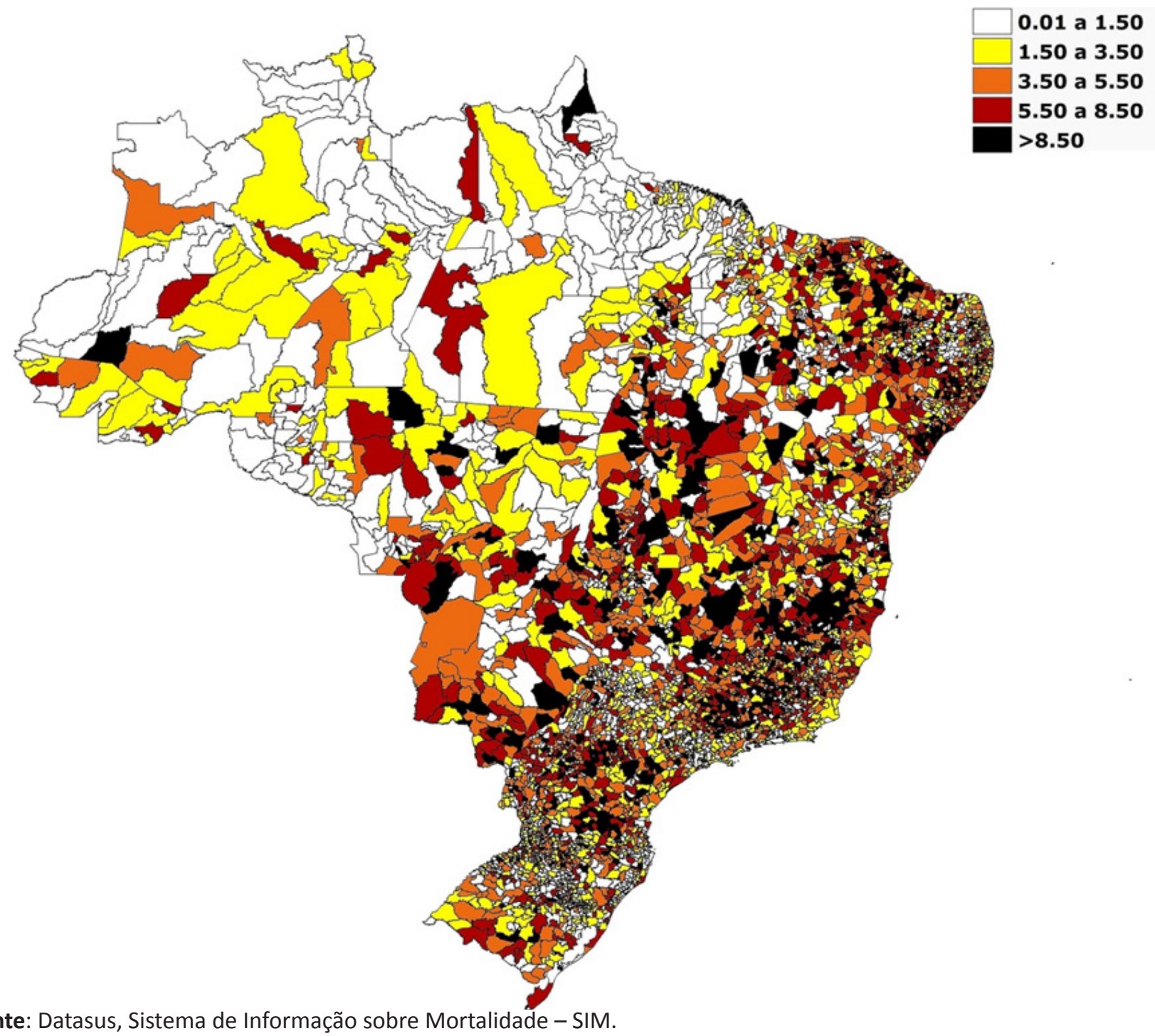

Na análise do BoxMap, observa-se a identificação/localização de áreas homogêneas constituídas por municípios com associação espacial, e com formação de aglomerados no Nordeste, Sudeste e Centro-Oeste do Brasil, aglutinando municípios cujos valores para a média das TMP são altas e cercados daqueles que também apresentam valores altos. Na região Norte, elevadas taxas em municípios do estado de Tocantins. Na região Nordeste, em municípios do Rio Grande do Norte; Ceará; Pernambuco, 
Sergipe e Bahia. Na região Sudeste, municípios dos estados de Minas Gerais e São Paulo e na região Centro-Oeste dos estados de Mato Grosso e Mato Grosso do Sul. Assim, o BoxMap destaca as áreas com prioridade para intervenção e/ou controle: as áreas de maior prioridade são os municípios agregados no quadrante Q1 (Alto/Alto); as áreas de menor prioridade são os municípios agregados no quadrante Q2 (Baixo/Baixo); e são áreas de prioridade intermediárias, os municípios agregados no quadrante Q3 (Alto/Baixo) e no quadrante Q4 (Baixo/Alto) (figura 2).

Figura 2. Box Map da taxa de mortalidade padronizada atribuível ao uso de álcool para o período de 2012 a 2016. Brasil, 2019.

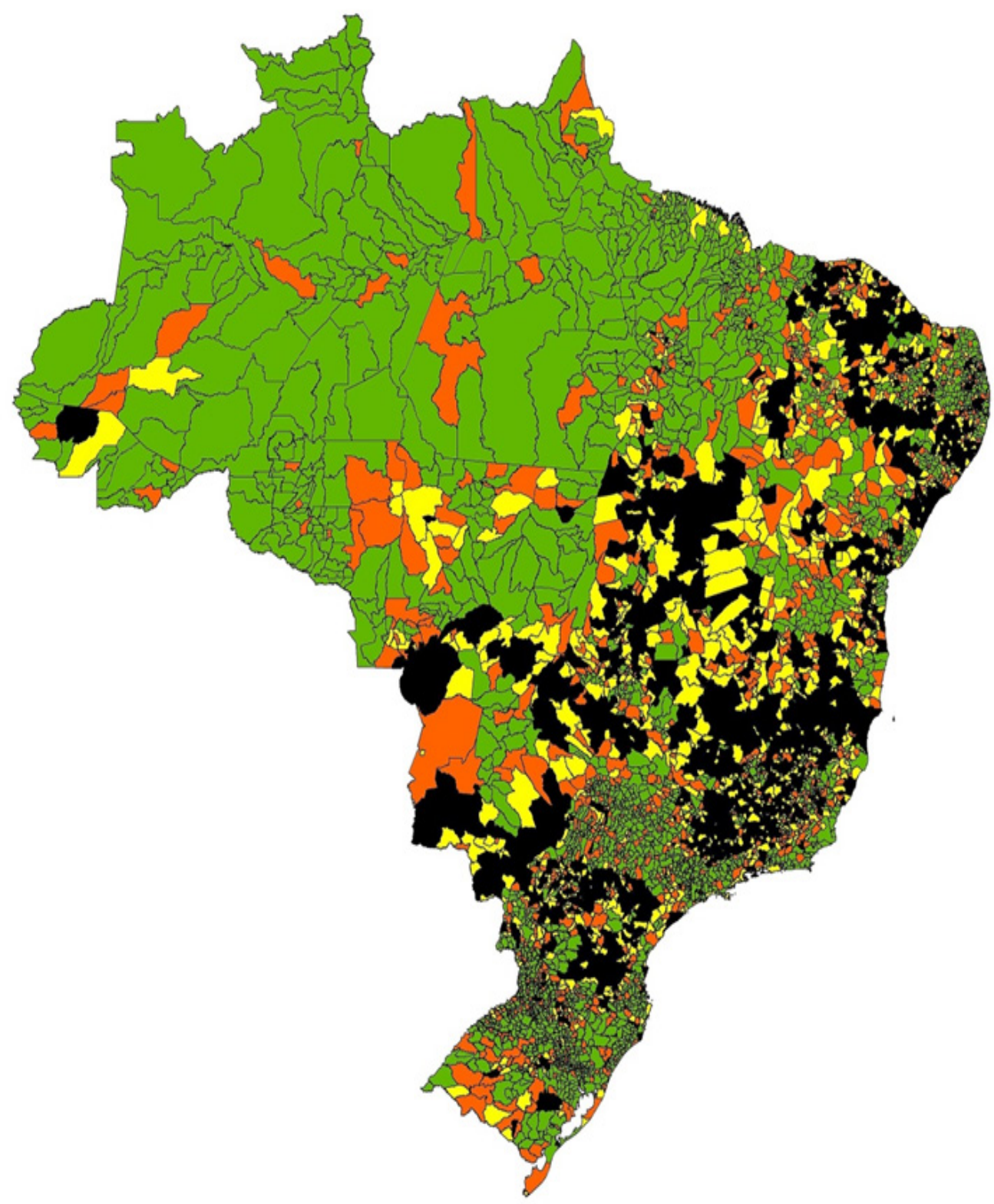

Alto/Alto
Baixo/Baixo
Alto/Baixo
Baixo/Alto

Fonte: Datasus, Sistema de Informação sobre Mortalidade - SIM.

O Índice de Moran Global obteve valor superior a zero e p-valor inferior a 0,05 para o período analisado, rejeitando a hipótese nula de aleatoriedade dos dados e concluindo que os municípios brasileiros seguem a tendência de agrupamentos, podendo-se assegurar que existe um padrão de dependência espacial na distribuição das taxas de mortalidade padronizadas.
O MoranMap da TMP mostra, na cor mais escura, as áreas consideradas significantes (>95\%). Evidenciou-se formação de clúster de alto/alto em municípios das regiões Nordeste, Sudeste e Centro-Oeste, enquanto foi verificada a presença de clúster de baixo/baixo nas regiões norte e sul do país (figura 3). 
Figura 3. Moran Map da taxa de mortalidade padronizada atribuível ao uso do álcool para o período de 2012 a 2016 . Brasil, 2019.

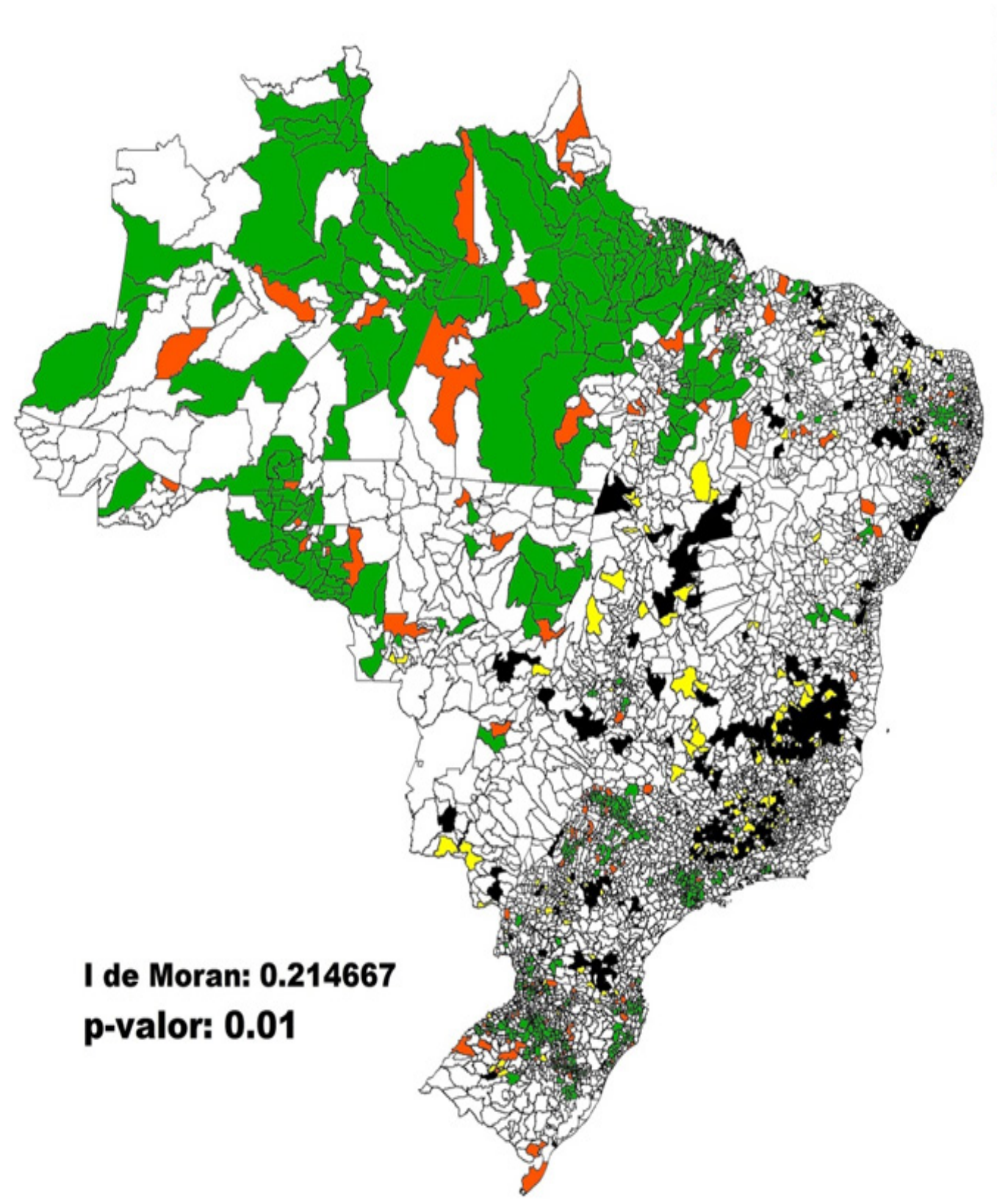

\begin{tabular}{|l|l}
\hline & Sem Significância \\
\hline Alto/Alto \\
Baixo/Baixo \\
\hline Alto/Baixo \\
Baixo/Alto
\end{tabular}

Fonte: Datasus, Sistema de Informação sobre Mortalidade - SIM.

A figura 4, representada pelo Lisa Map indica correlações espaciais locais significativas. Áreas nas cores preta e cinza escuro indicam autocorrelação espacial significativa positiva, enquanto áreas na cor cinza claro apresentam autocorrelação negativa, assim as áreas na cor branca são consideradas não significativas. O Lisa é usado para examinar os padrões de dados espaciais em uma escala de maior detalhe e permite a identificação de agrupamentos de objetos com valores de atributos semelhante. Observam-se "bolsões" de significância em municípios de estados das regiões Nordeste, Sudeste e Centro-Oeste. 
Figura 4. Lisa Map da taxa de mortalidade padronizada atribuível ao uso do álcool para o período de 2012 a 2016. Brasil, 2019.

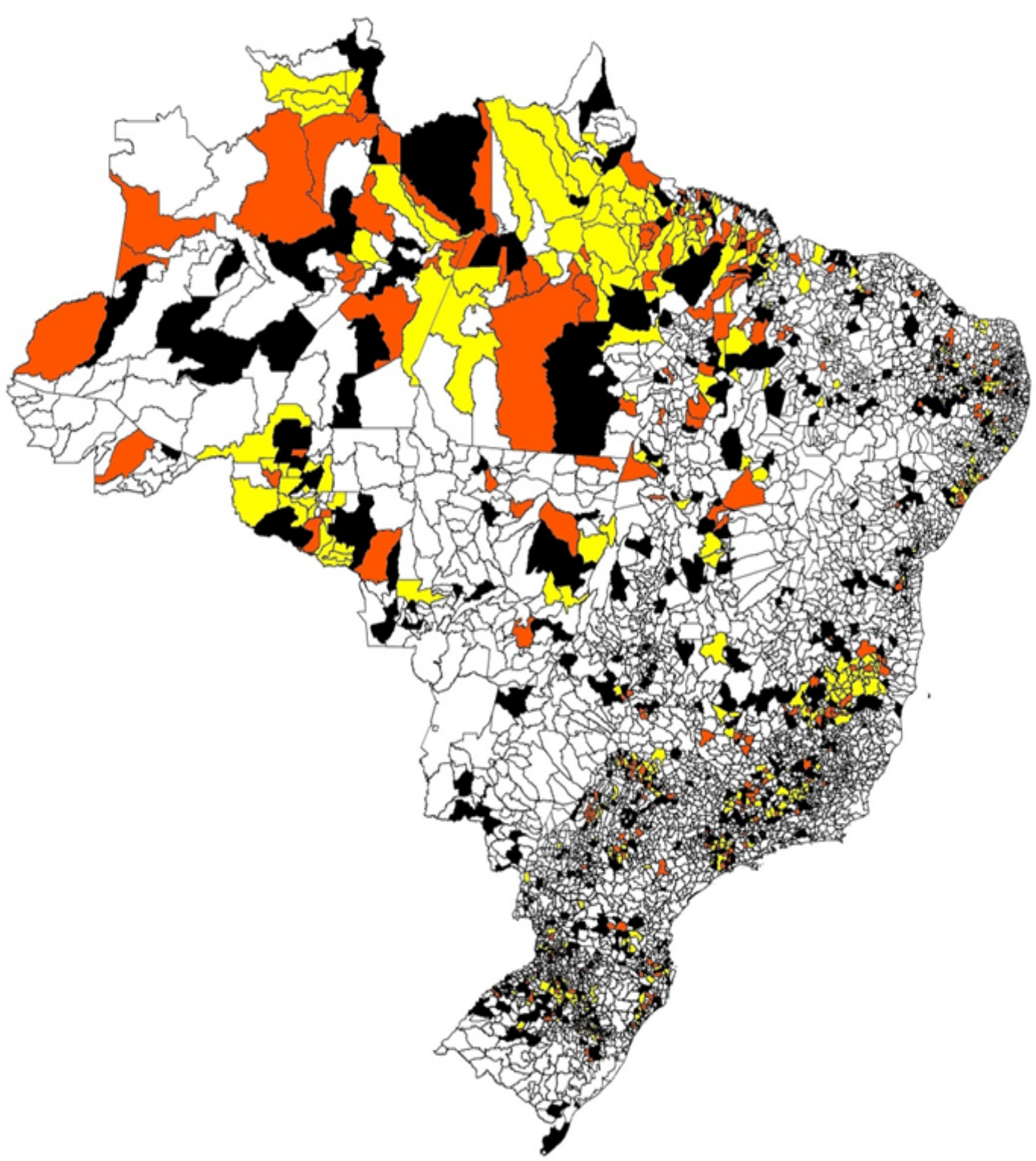

\begin{tabular}{|l|l}
\hline & Sem Significância \\
& Alto/Alto \\
Baixo/Baixo \\
Alto/Baixo \\
Baixo/Alto
\end{tabular}

Fonte: Datasus, Sistema de Informação sobre Mortalidade - SIM.

\section{DISCUSSÃO}

O estudo da distribuição espacial da mortalidade por transtornos mentais e comportamentais devido ao uso de álcool, registrados no Brasil, permitiu observar a presença de um padrão de correlação espacial para as taxas de mortalidade. Os resultados desse estudo apontam para diferenças na distribuição da mortalidade nas regiões do Brasil. Durante o período estudado, as maiores magnitudes das taxas de mortalidade no período de 2012 a 2016 foram observadas em algumas regiões do país, como Nordeste e Sudeste, mostrando, inclusive, formação de clusters alto-alto.

A partir dos dados analisados neste estudo, é possível perceber que, no Brasil, a cada ano, ocorre uma média de 6.633 óbitos atribuíveis ao uso de álcool. Nesse contexto, pesquisa com pessoas com transtornos decorrentes do consumo de substâncias psicoativas aponta que 63,1\% dos usuários apresentavam o álcool como substância de preferência e 95,7\%, utilizavam todos os dias. 25

A população masculina concentrou, aproximadamente, $90 \%$ dos óbitos, sendo esse evento predominante na faixa etária de 40 a 59 anos (56,39\%), em pessoas pretas e pardas $(60,17 \%)$, com escolaridade entre 1 a 3 anos de estudo (28,04\%), solteiros $(48,22 \%)$ e tendo o óbito ocorrido, principalmente, no hospital 
$(43,64 \%)$ e no domicílio $(42,66 \%)$ das vítimas.

O maior número de mortes entre os homens corrobora estudos anteriores acerca do tema, os quais apontam para a predominância desse público, tanto nas morbidades decorrentes do hábito de ingerir em demasia bebidas alcóolicas, como nos óbitos atribuíveis a esse comportamento nocivo. A explicação pode estar no maior consumo de álcool entre os homens, visto que dados da Pesquisa Nacional de Saúde (2013) mostraram que a prevalência do consumo abusivo de álcool é 3,3 vezes superior entre os homens em relação às mulheres, quando levada em consideração a população adulta (maiores de 18 anos) $)^{3,26}$.

Quanto à faixa etária, um estudo realizado no Brasil constatou que os números de óbitos por cirrose, câncer hepático e transtornos devidos ao uso de álcool cresceram 75\% quando observados os números de 1990 e de 2015, passando de 16.226 para 28.337 mortes no país. Ademais, revelou que, em 2015, a faixa etária que concentrou mais mortes por essas causas foi a de 50 a 59 anos (28,3\%), apresentando congruência com os dados desse estudo, o qual identificou a faixa etária de 40 a 59 anos como a que mais houve mortes atribuíveis ao uso de álcool. Ainda segundo o referido estudo comparativo, o pico da mortalidade por transtornos devidos ao uso de álcool ocorre, mais precocemente, na faixa dos 40-49 anos, em comparação com a cirrose (50-54 anos) e o câncer hepático, em geral a partir dos 65 anos $^{27}$

Esta pesquisa mostrou que $60 \%$ dos óbitos atribuíveis ao uso de álcool no País, no período analisado, concentraram-se em pessoas de cor preta e parda. Esse dado reforça outros estudos feitos sobre a temática, como uma pesquisa com óbitos por doenças, condições e lesões nas quais o consumo de álcool era causa necessária, revelando que que 56,6\% eram pessoas pretas/pardas ${ }^{3}$. Outro estudo com dados da Pesquisa Nacional de Saúde (2013) que analisou a prevalência de uso recente e excessivo episódico de álcool entre adultos, segundo características sociodemográficas, mostrou que 51,6\% da população total se constituiu de pessoas de cor de pele caracterizada como não branca ${ }^{26}$

Estudo sobre óbitos causados por doenças, condições e lesões, no triênio 2010 a 2012, mostrou que o consumo de álcool é a causa principal da morte. Constatou, ainda, que $62,6 \%$ dos óbitos atingiram pessoas com menos de 8 anos de escolaridade. Tal resultado assemelha-se com os dados desta pesquisa, na qual cerca de $66 \%$ dos óbitos envolveram pessoas cuja escolaridade era menor do que 8 anos de estudo. De acordo com o estudo citado, é provável que pessoas com maior escolaridade tenham mais acesso a serviços de saúde, assim como a diagnóstico e a tratamentos que evitariam potenciais danos ocasionados pelo álcool. Por sua vez, pessoas que possuem menos anos de escolaridade tendem a apresentar relação com outros fatores de risco sociais e ambientais, que podem agravar o quadro clínico desses sujeitos, vítimas do álcool ${ }^{3}$.

No tocante ao estado civil, os dados desse estudo apresentam-se consonantes com outras pesquisas realizadas no sentido de que o maior percentual de óbitos atribuíveis ao uso nocivo do álcool concentra-se em indivíduos solteiros. Um estudo que analisou 972 óbitos por causas "mortis" relacionadas ao transtorno de abuso e dependência de álcool em homens no ano de 2015, na Paraíba, verificou que $83,3 \%$ trataram de pessoas solteiras. Essas pesquisas mostram o risco aumentado de ser solteiro e vir ao óbito por uso abusivo do álcool, assim como evidenciam que uma relação estável ou casamento tornam-se fatores protetores para o mesmo mal ${ }^{26,28}$.

Consoante com outro resultado desta pesquisa, referente ao local de ocorrência dos óbitos, outros estudos apresentaram dados equivalentes. Um estudo que objetivou descrever a mortalidade relacionada ao álcool, no período de 1996 a 2007, em Ribeirão Preto (SP), constatou que a maior parte dos óbitos ocorreu no hospital ou no domicílio das vítimas.29 Outro estudo que buscou descrever os óbitos atribuíveis ao álcool em Sergipe, entre 1998 e 2010, também verificou predomínio de óbitos em ambiente hospitalar, seguido dos óbitos domiciliares ${ }^{30}$.

A ocorrência de óbitos atribuíveis ao uso nocivo do álcool no âmbito hospitalar ou de outros serviços de saúde é esperada em virtude das condições de saúde das vítimas. Já o alto percentual de óbitos, no contexto domiciliar, pode ser indicativo de que os indivíduos ou suas famílias, por desconhecerem sua condição de doente, estariam agindo com negligência quanto à assistência em saúde, o que torna os serviços de saúde responsáveis pela conscientização em prol do combate a estigmas e a preconceitos ${ }^{30}$.

Os resultados do presente estudo apresentaram "bolsões" de significância estatística em municípios das regiões Nordeste, Sudeste e Centro-Oeste. Por intermédio da análise exploratória espacial foi observado um comportamento de dependência espacial, nessas três regiões do país, com formação de cluster de alto/alto, ou seja, municípios com altas taxas de mortalidade atribuíveis ao uso de álcool se correlacionando no espaço com seus vizinhos que também apresentaram taxas extremas pela mesma causa de morte.

A esse respeito, observando as regiões brasileiras, um estudo, que analisou a mortalidade por dependência de álcool na população do país, utilizando a causa básica de morte das estatísticas oficiais de mortalidade no período de 1998 a 2002, constatou que os coeficientes de mortalidade por Dependência de Álcool (DA) das regiões do país mostraram-se maiores nas regiões Nordeste, Sudeste e Centro-Oeste, enquanto as regiões Norte e Sul apresentaram-se com menores números, o que é corroborado pelos dados desta pesquisa referente aos óbitos atribuíveis ao álcool ${ }^{31}$.

Em relação a essa variação regional, no tocante aos anos perdidos por morte prematura ou por incapacidade dos transtornos decorrentes do uso de álcool, estudos demonstram uma maior variação regional nas taxas de DALY no Ceará e no Sergipe (região Nordeste) que chegaram a quase quatro vezes 
maiores do que a do Pará (região Norte); já os transtornos decorrentes do uso de drogas apresentaram taxas homogêneas, em termos de proporção de DALY, em $2015^{32}$.

Sobre diferenciais regionais, um estudo intitulado "Persistência das desigualdades regionais no Brasil: polarização e divergência" demonstra que, nos subconjuntos Norte/Nordeste e resto do país, há maior identificação dos estados e municípios dentro de cada grupo (pobres com pobres e ricos com ricos), e um aumento da distância entre eles, apontando para desigualdades, cada vez mais crescente, dentro do mesmo país ${ }^{33}$.

Estudos apontam que o uso de drogas como álcool e o fumo pode despertar para o uso de outras substâncias consideradas ilícitas. Conforme achados em um estudo observacional no Centro de Atenção Psicossocial para usuários de álcool e outras drogas (CAPSad) do município de Teresina - Piauí, em geral, os participantes iniciaram o uso de crack, após uma sequência de uso de outras substâncias como álcool e tabaco; caracterizaram, assim, uma situação de poliuso de substâncias químicas ${ }^{34}$.

Assim, o uso abusivo do álcool e outras drogas pode acarretar situações conflitos intrafamiliares devido aos problemas financeiros desencadeados pelo uso de recursos para manter o vício. Em um estudo em Santos - SP, a maior parte da fonte de renda dos usuários era proveniente de atividades informais, e a média de tempo de vínculo formal de trabalho foi de quatro anos, visto que, nos últimos 30 dias, o número de dias trabalhados foi em média nove dias ${ }^{16}$. São situações de sobrecargas vivenciadas por familiares de indivíduos dependentes químicos em que as famílias são afetadas pelas implicações psicossociais do usuário e pelos prejuízos financeiros para a manutenção do consumo de drogas, sejam lícitas ou não ${ }^{20}$.

É premente a necessidade de políticas públicas que atentem para os cuidados com o usuário. Uma pesquisa realizada em 2013 em um CAPS III, em Minas Gerais, mostrou alta frequência de comportamento suicida19. Outro estudo evidenciou que, entre os motivos da tentativa de suicídio expressos por homens usuários de álcool e outras drogas, a ação suicida tem uma relação direta no contexto social dos sujeitos ${ }^{35}$.

Em uma observação sobre drogas psicoativas entre adolescentes do Rio Grande do Sul, os jovens relataram o primeiro contato com as drogas na casa de amigos/conhecidos e que, a maioria das vezes, as drogas foram oferecidas por desconhecidos. Ainda relataram alterações nos níveis de impulsividade e agressividade após a utilização do álcool e/ou outras drogas ${ }^{36}$

A relação entre o uso do álcool, outras drogas e os eventos acidentais ou situações de violência evidencia o aumento na gravidade das lesões e a diminuição dos anos potenciais de vida da população, expondo as pessoas a comportamentos de risco ${ }^{37}$.

É necessário o fortalecimento das ações de prevenção relacionadas ao uso do álcool e outras substâncias psicoativas no Brasil. A dependência do álcool no Brasil, em 2016, esteve associada a $69,5 \%$ e $42,6 \%$ dos índices de cirrose hepática, a $36,7 \%$ e $23 \%$ dos acidentes de trânsito e a $8,7 \%$ e $2,2 \%$ dos índices de câncer - respectivamente, entre homens e mulheres. Especificamente sobre os transtornos relacionados ao uso do álcool, estima-se que 4,2\% (6,9\% entre homens e 1,6\% entre mulheres) dos brasileiros preenchem critérios para abuso ou dependência ${ }^{21}$.

O contexto familiar e sociocultural em que o alcoolista está inserido, assim como os programas educativos se defrontam com a aceitação cultural da bebida e com o poder cultural e político da mídia que explora essa aceitação social, associando as bebidas à sensualidade, ao sucesso, obscurecendo os problemas do beber sem limites ${ }^{38}$.

Sendo o uso nocivo do álcool um grave problema de saúde pública com consequências negativas para o indivíduo, para a família e para a sociedade, faz-se necessário que as políticas públicas tenham uma maior abrangência, com suas reformulações para além do campo da saúde, com planejamento e ações intersetoriais de forma a priorizar os grupos mais vulneráveis.

\section{CONCLUSÃO}

É possível perceber que a mortalidade por causas atribuíveis ao uso de álcool está desigualmente distribuída no Brasil e não necessariamente está relacionada as piores condições de vida, uma vez que foi possível observar a presença de clusters de altas taxas de mortalidade em regiões socioeconomicamente desenvolvidas, como o Sudeste do País.

Os achados fortalecem a necessidade de se buscarem explicações para as diferenças dos índices de óbitos por transtornos mentais e comportamentais atribuídos ao uso de álcool em seu contexto local, reforçando a importância da organização da vigilância epidemiológica e a análise focada nos municípios de maior ocorrência, de forma a favorecer uma compreensão desse problema de saúde pública, e melhorar as possibilidades de prevenção.

No Brasil, fica evidente que seus municípios seguem a tendência de agrupamentos e um padrão de dependência espacial na distribuição das taxas de mortalidade padronizadas, atribuídas ao uso de álcool.

As técnicas utilizadas mostraram-se úteis para a identificação de agrupamentos contínuos (clusters), de altos e baixos valores, áreas de transição entre clusters. Também foi possível avaliar os agrupamentos quanto ao nível de significância da associação espacial, selecionando os agrupamentos mais importantes presentes nas regiões Nordeste, Sudeste e Centro-Oeste do Brasil.

O conjunto de ferramentas exploratórias utilizadas neste estudo possibilitaram a exploração de dados espaciais, na identificação de municípios com altas taxas e seus vizinhos com situação 
igual, o que pode ser um bom diagnóstico para o planejamento, criação ou ampliação de políticas intra e intersetoriais para o enfrentamento do uso abusivo do álcool e problemas relacionados.

A limitação desse estudo está relacionada à utilização de dados secundários sobre mortalidade que está sujeito ao subregistro, apesar de, nos últimos anos, reconhecer-se que o Sistema de Informação sobre Mortalidade (SIM) no Brasil obteve ganhos significativos tanto na qualidade do preenchimento da declaração de óbito, como no registro no sistema.

As contribuições deste estudo, por sua vez, referem-se aos subsídios gerados para políticas públicas com ênfase nas ações geograficamente determinadas, com políticas orientadas para a priorização de municípios, estados e regiões que apresentam altas taxas de mortalidades atribuíveis ao uso do álcool.

\section{REFERÊNCIAS}

1. Institute for Health Metrics and Evaluation. Global burden of disease country profiles [Internet]. Seattle: IHME; 2013 [acesso 2019 Ago 4]. Disponível em: http://www.healthmetricsandevaluation.org/gbd/country-profiles.

2. Organização Mundial de Saúde. Stratégie mondiale visant à réduire l'usage nocif de l'alcool. Genebra: Organização Mundial de Saúde; 2010. 48 p.

3. Garcia LP, Freitas LRS, Gawryszewski VP, Duarte EC. Uso de álcool como causa necessária de morte no Brasil, 2010 a 2012. Rev Panam Salud Publica [Internet]. 2015 Set [acesso 2019 Ago 10]; 38(5): 418-24. Disponível em: https://www. scielosp.org/pdf/rpsp/2015.v38n5/418-424/pt.

4. Veiga LDB, Santos VC, Santos MG, Ribeiro JF, Amaral ASN, Nery AA, et al . Prevalência e fatores associados à experimentação e ao consumo de bebidas alcoólicas entre adolescentes escolares. Cad. saúde colet [Internet]. 2016 Set [acesso 2019 Ago 16]; 24(3): 368-375. Disponível em: http://www.scielo.br/ scielo.php?script=sci_arttext\&pid=S1414-462X2016000300368\&Ing=en. doi: http://dx.doi.org/10.1590/1414-462×201600030037.

5. Damacena GN, Malta DC, Boccolini CS, Souza PRB Júnior, Almeida WS, Ribeiro LS, et al. Consumo abusivo de álcool e envolvimento em acidentes de trânsito na população brasileira, 2013. Ciênc. saúde coletiva [Internet]. 2016 Dez [citado em 14 Ago 2019]; 21(12): 3777-3786. Disponível em: http://www.scielo.br/ scielo.php?script=sci_arttext\&pid=S1413-81232016001203777\&\&ng=pt. doi: http://dx.doi.org/10.1590/1413-812320152112.25692015.

6. World Health Organization. Global status report on alcohol and health 2014 Geneva: WHO; 2014.

7. Mangueira SO, Guimarães FJ, Mangueira, JO, Fernandes AFC, Lopes MVO. Promoção da saúde e políticas públicas do álcool no Brasil: revisão integrativa da literatura. Psicologia \& Sociedade [Internet]. 2015 [acesso 2019 Ago 02] 27(1):157-68. Disponível em: <http://www.scielo.br/scielo.php?script=sci arttext\&pid=S0102-71822015000100157\&Ing=en\&nrm=iso>. doi: http:// dx.doi.org/10.1590/1807-03102015v27n1p157.

8. Malta DM, Sardinha LMV, Mendes I, Barreto SM, Giatti L, Castro IRRD, et al. Prevalência de fatores de risco e proteção de doenças crônicas não transmissíveis em adolescentes: resultados da Pesquisa Nacional de Saúde do Escolar (PeNSE), Brasil, 2009. Ciênc. saúde coletiva [Internet]. 2010 Out [acesso 2019 Ago 12]; 15(Suppl 2): 3009-3019. Disponível em: http://www.scielo.br/ scielo.php?script=sci arttext\&pid=S1413-81232010000800002\&lng=pt. doi: http://dx.doi.org/10.1590/\$1413-81232010000800002.

9. Roerecke M, Rehm J. Cause-specific mortality risk in alcohol use disorder treatment patients: a systematic review and meta-analysis. Int. J. Epidemiol. 2014; 43(3): 906-919. Disponível em: http://ije.oxfordjournals.org/ content/43/3/9 06.full.pdf+html

10. World Health Organization. World health statistics. Geneva: WHO; 2012.

11. World Health Organization. Global status report on alcohol and health. Geneva: WHO; 2011.

12. Ministério da Saúde (BR). Portaria no 2197, de 04 outubro. de 2004. Redefine e amplia a atenção integral para usuários de álcool e outras drogas, no âmbito do Sistema Único de Saúde - SUS, e dá outras providências [Internet].
Diário Oficial [da] República Federativa do Brasil. 2004 Out 05 [acesso 2019 Ago 15]. Disponível em: http://bvsms.saude.gov.br/bvs/saudelegis/gm/2004/ prt2197_14_10_2004.html.

13. Brasil. Decreto no 6.117, de 22 maio de 2007. Aprova a política nacional sobre o álcool, dispõe sobre as medidas para redução do uso indevido de álcool e sua associação com a violência e criminalidade, e dá outras providências [Internet]. Diário Oficial [da] República Federativa do Brasil. 2007 Maio 23 [acesso 2019 Ago 10]. Disponível em: http://www.planalto.gov.br/ccivil_03/ ato2007-2010/2007/decreto/d6117.htm.

14. Ministério da Saúde (BR).. Portaria no 1190, de 04 junho de 2009. Institui o plano emergencial de ampliação do acesso ao tratamento e prevenção em álcool e outras drogas no Sistema Único de Saúde (SUS) (PEAD 2009-2010) e define suas diretrizes gerais, ações e metas [Internet]. Diário Oficial [da] República Federativa do Brasil. 2019 Jun 05 [acesso em 2019 Ago 15]. Disponível em: http://bvsms.saude.gov.br/bvs/saudelegis/gm/2009/prt1190_04_06_2009. html.

15. Ministério da Saúde (BR). Portaria no 3.088, de 23 dezembro de 2011. Institui a rede de atenção psicossocial para pessoas com sofrimento ou transtorno mental e com necessidades decorrentes do uso de crack, álcool e outras drogas, no âmbito do Sistema Único de Saúde (SUS) [Internet]. Diário Oficial [da] República Federativa do Brasil. 2011 Dez 23 [acesso em 2019 Ago 15]. Disponível em: http://bvsms.saude.gov.br/bvs/saudelegis/gm/2011/ prt3088_23_12_2011_rep.html.

16. Malta DC, Silva JB Júnior. O Plano de Ações Estratégicas para o Enfrentamento das Doenças Crônicas Não Transmissíveis no Brasil e a definição das metas globais para o enfrentamento dessas doenças até 2025: uma revisão. Epidemiol. Serv. Saúde [Internet]. 2013 Mar [acesso 2019 Ago 02]; 22(1): 151-164. Disponível em: http://scielo.iec.gov.br/scielo.php?script=sci_ arttext\&pid=S1679-49742013000100016\&lng=pt. doi: http://dx.doi. org/10.5123/S1679-49742013000100016.

17. Dázio EMR, Zago MMF, Fava SMCL. Uso de álcool e outras drogas entre universitários do sexo masculino e seus significados. Rev. esc. enferm. USP [Internet]. 2016 Out [acesso em 2019 Ago 16]; 50(5): 785-791. Disponível

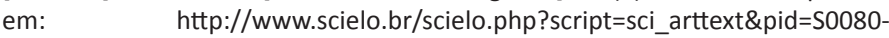
62342016000500785\&lng=pt. doi: http://dx.doi.org/10.1590/s0080623420160000600011 .

18. Sanches ACF, Souza LGS. Consumo de álcool, características autoatribuídas de gênero e qualidade de vida em homens de classe popular, usuários de Unidade de Saúde da Família. Estud. psicol. (Natal) [Internet]. 2016 Set [acesso 2019 Ago 04]; 21(3): 261-271. Disponível em: http://www.scielo.br/scielo. php?script=sci arttext\&pid=S1413-294X2016000300261\&Ing=en\&nrm=iso. doi: http://dx.doi.org/10.5935/1678-4669.20160025.

19. Ministério da Justiça e Segurança Pública (BR). Secretaria Nacional de Políticas sobre Drogas. Prevenção dos problemas relacionados ao uso de drogas: capacitação para conselheiros e lideranças comunitárias. 6. ed. Brasília; 2014.

20. Soccol, KLS; Terra MG, Ribeiro DB, Mostardeiro SCTS, Teixeira JKS, Souto VTS, et al. Sobrecarga financeira vivenciada por familiares cuidadores de indivíduos dependentes químicos. Rev Enferm UFSM [Internet]. 2014 Jul-Set [acesso 2019 Agosto 9]; 4 (3): [cerca de 10 p.]. Disponível em: https://doi. 
org/10.5902/2179769211264.

21. Organização Mundial da Saúde. Relatório Global sobre Álcool e Saúde 2018. Genebra: OMS; 2019.

22. Meloni, JN; Laranjeira, R. Custo social e saúde no consumo de álcool. Rev. Bras. Psiquiatr. [Internet]. 2004 Maio [acesso 2019 Nov 03]; 26(Supl. 1): 7-10. Disponível em: http://www.scielo.br/scielo.php?script=sci_arttext\&pid=S151644462004000500003\&lng=en. doi: http://dx.doi.org/10.1590/S1516 44462004000500003.

23. Anselin L. Local indicators of spatial association - LISA. Geogr Anal. 1995; 27(2): 93-115.

24. Câmara, G; Carvalho, MS; Cruz, OG; Correa, V. Análise Espacial de Áreas. São José dos Campos-SP: INPE; 2002.

25. Oliveira VC, Capistrano FC, Ferreira ACZ, Kalinke LP, Felix JVC, Maftum MA. Perfil sociodemográfico e clínico de pessoas atendidas em um CAPS AD do sul do Brasil. Rev baiana enferm [Internet]. 2017 [acesso 2019 Ago 9]; 31(1): [cerca de 12 p.]. Disponível em: https://portalseer.ufba.br/index.php/enfermagem/ article/view/16350/pdf. doi: https://doi.org/10.18471/rbe.v31i1.16350

26. Machado IE, Monteiro MG, Malta DC, Lana FCF. Pesquisa Nacional de Saúde 2013: relação entre uso de álcool e características sociodemográficas segundo o sexo no Brasil. Rev. bras. epidemiol [Internet]. 2017 Jul [acesso 2019 Ago 3]; 20(3): 408-422. Disponível em: http://www.scielo.br/scielo. php?script=sci_arttext\&pid=S1415-790X2017000300408\&lng=en. doi: http:// dx.doi.org/10.1590/1980-5497201700030005.

27. MeloAPS, França EB, Malta DC, Garcia LP, Mooney M, Naghavi M. Mortalidade por cirrose, câncer hepático e transtornos devidos ao uso de álcool: Carga Global de Doenças no Brasil, 1990 e 2015. Rev Bras Epidemiol [Internet]. 2017 Maio [acesso 2019 Ago 5]; 20(Suppl 1):61-74. Disponível em: http://www.scielo.br/ scielo.php?script=sci_arttext\&pid=S1415-790X2017000500061\&Ing=en. doi: http://dx.doi.org/10.1590/1980-5497201700050006.

28. Silva JVS. Estudo da mortalidade associado ao transtorno de abuso e dependência ao álcool. Santos/SP. [dissertação]. Santos (SP): Universidade Católica de Santos; 2017.

29. Benedicto RP. Causas múltiplas de morte relacionadas ao consumo de álcool na microrregião de Ribeirão Preto-SP, 1996-2007. [dissertação]. Ribeirão Preto (SP): Escola de Enfermagem de Ribeirão Preto; 2011.

30. Bohland AK, Gonçalves AR. Mortalidade atribuível ao consumo de bebidas alcoólicas. SMAD, Rev. Eletrônica Saúde Mental Álcool Drog. (Ed. port.) [Internet]. 2015 Set [acesso 2019 Ago 9]; 11(3):136-144. Disponível em: http://pepsic.bvsalud.org/scielo.php?script=sci_arttext\&pid=S180669762015000300004\&lng=pt\&nrm=iso. doi: http://dx.doi.org/10.11606/ issn.1806-6976.v11i3p136-144.

31. Marín-León L, Oliveira HB, Botega NJ. Mortalidade por dependência de álcool no Brasil: 1998 - 2002. Psicol. estud. [Internet] 2007 Abr [acesso 2019 Ago 10]; 12(1): 115-121. Disponível em: http://www.scielo.br/scielo. php?script=sci_arttext\&pid=S1413-73722007000100014\&Ing=en\&nrm=iso. doi: http://dx.doi.org/10.1590/S1413-73722007000100014.

32. Bonadiman, CSC, Passos VMA, Mooney M, Naghavi M, Melo APS. A carga dos transtornos mentais e decorrentes do uso de substâncias psicoativas no Brasil: Estudo de Carga Global de Doença, 1990 e 2015. Rev. bras. epidemiol [Internet]. 2017 [acesso 2019 Ago 9]; 20(suppl. 1): 191-204. Disponível em: https://doi. org/10.1590/1980-5497201700050016. doi: https://doi.org/10.1590/19805497201700050016.

33. Figueiredo E, Porto S. Junior. Persistência das desigualdades regionais no Brasil: polarização e divergência. Nova Econ. 2015 Abr. 25(1):195-208. doi: http://dx.doi.org/10.1590/0103-6351/2106.

34. Carvalho NAR, Santos JDM, Silva JFG Junior, Monteiro CFS, Lima LAA. Perfil de usuários de crack. Rev Enferm UFPI. [Internet]. 2015 Apr-Jun [acesso: 2019 Agosto 15]; 4(2 [cerca de 7 p.]. Disponível em: https://revistas.ufpi.br/index. php/reufpi/article/view/3554. doi: https://doi.org/10.26694/reufpi.v4i2.3554.

35. Ribeiro DB, Terra MG, Soccol KLS, Schneider JF, Camillo LA, Plein FAZ. Motivos da tentativa de suicídio expressos por homens usuários de álcool e outras drogas. Rev. Gaúcha Enferm. [Internet]. 2016 [acessi 2019 Ago 4]; 37(1): [cerca de 8 p.]. Disponível em: http://www.scielo.br/scielo.php?pid=S198314472016000100414\&script=sci_abstract\&tlng=pt. doi: http://dx.doi. org/10.1590/1983-1447.2016.01.54896.

36. Almeida RMM, Trentini LB, Klein LA, Macuglia GR, Hammer C, Tesmmer M. Uso de Álcool, Drogas, Níveis de Impulsividade e Agressividade em Adolescentes do Rio Grande do Sul. Psico [Internet]. 2014 Jan.-Mar. [acesso 2019 Ago 15]; 45(1): 65-72. Disponível em: http://revistaseletronicas.pucrs.br/ revistapsico/ojs/index.php/revistapsico/article/view/12727. doi: http://dx.doi. org/10.15448/1980-8623.2014.1.12727.

37. Ministério da Saúde (BR). Secretaria Executiva. Coordenação Nacional de DST e Aids. A Política do Ministério da Saúde para atenção integral a usuários de álcool e outras drogas. Brasília: Ministério da Saúde; 2003.

38. Acselrad G (Org.). Consumo do álcool no Brasil. Série Cadernos FLACSO, Número 12, FLACSO Brasil, 2014.

\section{Como citar este artigo/How to cite this article:}

Marques MV, Silva DN Júnior, Santos EGO, Santos SSA, Neves SMB, Amador AE. Distribuição espacial das mortes atribuíveis ao uso de álcool no Brasil. J Health Biol Sci. 2020; 8(1):1-11 